\title{
Suppression of Nonlinear Distortion in Few-Mode Fibres using Strong Mode Coupling
}

\author{
Filipe M. Ferreira, Naoise Mac Suibhne, Christian Sánchez, Stylianos Sygletos, Andrew Ellis \\ Aston Institute of Photonic Technologies, Aston University, Birmingham, B4 7ET, UK \\ f.ferreira@aston.ac.uk
}

\begin{abstract}
In this paper, we investigate the impact of linear mode coupling on the efficiency of intermodal four-wave mixing and on the group delay statistics in few-mode fibres. The investigation will include not only the weak or strong linear coupling regimes, but also the transition region between them, the intermediate coupling regime. This analysis will allow to assess the level of coupling strength require to suppress the nonlinear distortion in a few-mode fibre below the level of distortion for single-mode propagation without mode coupling.
\end{abstract}

Keywords-Few-mode fibres, linear mode coupling, intermodal four-wave mixing.

\section{INTRODUCTION}

Mode-division multiplexing over few-mode fibres (FMFs) has been proposed as a next-generation solution to overcome the impeding installed capacity exhaustion of current single-mode fibres (SMFs). In principle, an $N$-fold capacity increase can be obtained by using one FMF guiding $N$ independent modes in place of one single-mode fibre (SMF). However, FMFs present new impairments that have to be addressed in order to reach their full capacity [1]-[4], namely: linear mode coupling, differential mode delay, and intermodal nonlinear effects. Among these impairments, linear coupling plays a key role since stronger coupling acts to lower the overall group delay spread [2] and the intermodal nonlinear distortion [5]. However whilst nonlinear intermodal interactions have been experimentally measured [3] and numerical models have been made available [4]-[8], the knowledge about the nature of the dependence of the nonlinear distortion on the coupling strength is not fully understood in the intermediate regime.

In this paper, we review and extend our most recent results on impact of linear mode coupling on the intermodal nonlinear interaction efficiency [9] and on the group delay statistics [10]. Section II reviews the generalization of the single-mode fourwave mixing (FWM) theory to a multi-mode fibre. Section III presents the numerical model developed for linear mode coupling in FMFs. Section IV reviews the impact of linear mode coupling on the statistics of mode group delays. Finally, Section $\mathrm{V}$ evaluates the dependence of the intermodal nonlinear interaction efficiency on the linear mode coupling strength through exhaustive stepwise simulation [7]-[9], considering a particular FMF supporting four non-degenerate linearly polarized (LP) modes for simplicity. In particular our simulations shown that with linear mode coupling beyond $-20 \mathrm{~dB} / 100 \mathrm{~m}$ a suppression of nonlinear distortion below the isolated mode without linear mode coupling can be achieved.

\section{INTERMODAL NONLINEAR INTERACTIONS}

The commercial use of FMF in long haul systems is likely to require the use of a substantial fraction of the bandwidth. In such case, the number of intermodal nonlinear interactions that are velocity-matched increases significantly, especially in fibres with low differential mode delay (required for low complexity digital signal processing). For FMFs, velocity matching may be achieved by balancing the two dispersive processes present, the differential mode delay and the chromatic dispersion [3].

The generalization of the single-mode four-wave mixing (FWM) theory to a multi-mode fibre has been shown to be straightforward [4]-[6]. When considering three waves denoted $p, q, r$ propagating in modes denoted $a, b, c$, respectively, the nonlinear signal field $E_{d s}$ generated at angular frequency $\omega_{s}=$ $\omega_{p}+\omega_{q}-\omega_{r}$ in mode $d$ is:

$E_{d s}=\gamma_{a b c d} E_{a p} E_{b q} E_{c r}^{*} \frac{1-e^{-\left(\alpha+\mathrm{j} \Delta \beta_{a b c d, p q r s}\right) L}}{\mathrm{j} \Delta \beta_{a b c d, p q r s} L+\alpha} e^{-\left(\alpha / 2+\mathrm{j} \beta_{d s}\right) L}$

where $\alpha$ is the attenuation, $L$ is the span length, $\gamma_{a b c d}$ is the nonlinear coefficient for the interaction between modes $a, b, c$, $d$, and $\Delta \beta_{a b c d, p q r s}$ is the phase mismatch between waves $p, q$, $r$, s. The phase mismatch is given by $\Delta \beta_{a b c d, p q r s}=\beta_{a p}+$ $\beta_{b q}-\beta_{c r}-\beta_{d s}$ where $\beta_{a p}$ is the propagation constant of mode $a$ at angular frequency $\omega_{p}$.

For an optical super-channel with a total bandwidth $B$ the total nonlinear noise generated by FWM between a given set of modes may be calculated by integrating the product of (1) with the signal power spectral density (PSD) in each mode. A closed form solution for this integral was obtained (and experimentally validated) for the case of a signal with a rectangular spectrum (OFDM or Nyquist WDM super channel) in each interacting mode, and the overall efficiency parameter $\eta_{a b c d}$ was shown to be [12]:

$$
\begin{gathered}
\eta_{a b c d}=\frac{\gamma_{a b c d}^{2}}{\pi \alpha\left|\beta^{(2)}\right|}\left[\ln \left(\frac{B^{2}+2 B \Delta f_{a b c d}}{2 f_{w}}\right)+s \ln \left(s \frac{B^{2}-2 B \Delta f_{a b c d}}{2 f_{w}}\right)\right], \\
\text { with: } \\
\Delta f_{a b c d}=\left(\beta_{a}^{(1)}+\beta_{b}^{(1)}-\beta_{c}^{(1)}-\beta_{d}^{(1)}\right) / 2 \pi \beta^{(2)} ; \\
s=\operatorname{sign}\left(B-2 \Delta f_{a b c d}\right) ; f_{w}=\sqrt{\alpha / 4 \pi^{2}\left|\beta^{(2)}\right|}
\end{gathered}
$$




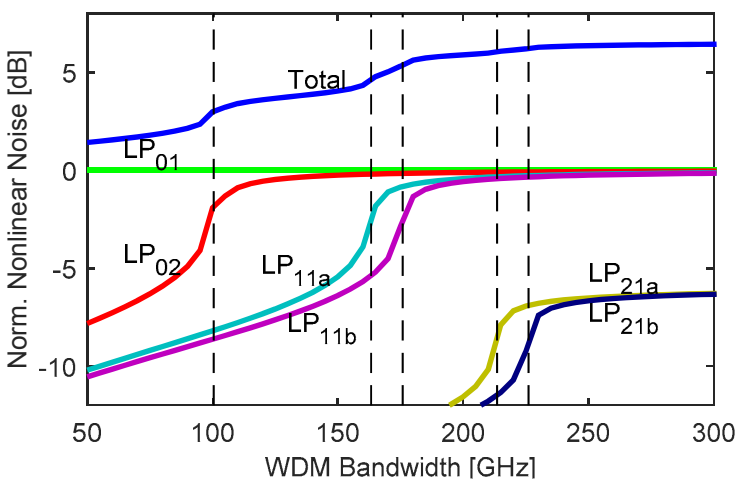

Fig. 1. Contribution to the nonlinear noise power in the $\mathrm{LP}_{01}$ mode for signals propagating in higher order modes normalized by the $\mathrm{LP}_{01}$ intramodal nonlinear noise power, as a function of WDM bandwidth.

TABLE I. CHARACTERISTICS OF THE FOUR LP MODE FMF [18].

Attenuation [dB/km]: 0.2
\begin{tabular}{|l|r|r|r|r|r|r|}
\hline & Differential Mode Delay $[\mathrm{ps} / \mathrm{km}]$ \\
\hline $\mathrm{LP}_{01}$ & 0 & 8 & 13 & 14 & 17 & 18 \\
\hline
\end{tabular}

Chromatic Dispersion $[\mathrm{ps} / \mathrm{nm} / \mathrm{km}]$

$\mathrm{LP}_{01}, \mathrm{LP}_{02}, \mathrm{LP}_{11 \mathrm{a}}, \mathrm{LP}_{11 \mathrm{~b}}, \mathrm{LP}_{21 \mathrm{a}}, \mathrm{LP}_{21 \mathrm{~b}}: 20$

Effective Area $\left[\mu^{2}{ }^{2}\right]$

\begin{tabular}{|l|c|c|c|c|c|c|}
\hline & $\mathrm{LP}_{01}$ & $\mathrm{LP}_{02}$ & $\mathrm{LP}_{11 \mathrm{a}}$ & $\mathrm{LP}_{11 \mathrm{~b}}$ & $\mathrm{LP}_{21 \mathrm{a}}$ & $\mathrm{LP}_{21 \mathrm{~b}}$ \\
\hline $\mathrm{LP}_{01}$ & 146 & 291 & 291 & 291 & 583 & 583 \\
\hline $\mathrm{LP}_{02}$ & 291 & 290 & 578 & 578 & 578 & 578 \\
\hline $\mathrm{LP}_{11 \mathrm{a}}$ & 291 & 578 & 193 & 579 & 386 & 386 \\
\hline $\mathrm{LP}_{11 \mathrm{~b}}$ & 291 & 578 & 579 & 193 & 386 & 386 \\
\hline $\mathrm{LP}_{21 \mathrm{a}}$ & 583 & 578 & 386 & 386 & 257 & 772 \\
\hline $\mathrm{LP}_{21 \mathrm{~b}}$ & 583 & 578 & 386 & 386 & 772 & 257 \\
\hline
\end{tabular}

where $\beta_{a}^{(1)}$ is the group delay of mode $a, \beta^{(2)}$ is the second order dispersion coefficient, and $\Delta f_{a b c d}$ is the velocity-matched frequency offset. All modes are assumed to have identical second order dispersion coefficients $\beta^{(2)}$ and frequency independent mode group velocities $\beta_{a}^{(1)}$. Note that the FWM efficiency is maximized at a velocity-matched frequency offset $\Delta f_{a b c d}$ where chromatic dispersion and differential mode delay cancel exactly. Since it is also necessary to cancel the contributions due to $\beta_{a}^{(0)}$ in this paper strong intermodal phasematching is only considered for interactions of pairs of modes $(a=d, b=c$ or $b=d, a=c)$ [13]. Finally, the total nonlinear power generated in mode $d$ is given by $\left(\sum_{a, b, c} \eta_{a b c d}\right) P_{a} P_{b} P_{c}$, where $P_{a}$ is the signal power spectral density in mode $a$.

Fig. 1 shows the nonlinear noise power generated at the centre of the WDM signal as a function of the overall bandwidth for a particular four LP mode fibre with no linear mode coupling. The fibre considered has a graded-index core with a refractive index relative difference of $4.5 \times 10^{-3}$ and a radius of $12.83 \mu \mathrm{m}$, optimized in [18] for low DMD. The fibre characteristics are presented in Table 1. In Fig. 1, a number of discontinuities are apparent in the total nonlinear noise, in addition to the logarithmically increasing background expected for a SMF [19].

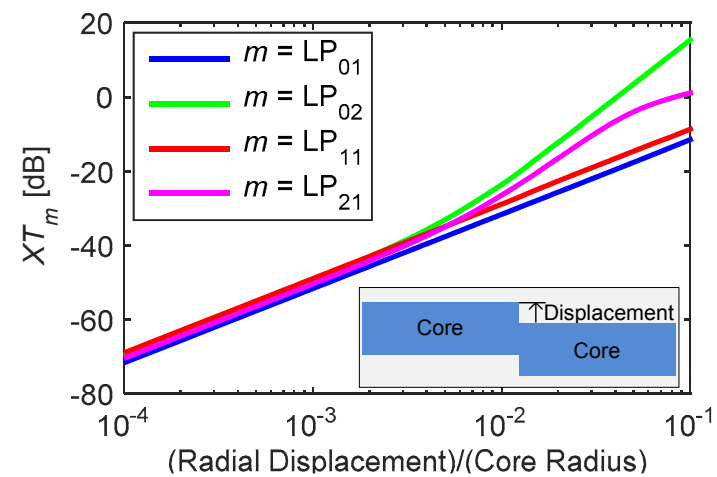

Fig. 2. $X T_{m}$ averaged over the displacement azimuth as a function of the radial displacement

These discontinuities correspond to the signal bandwidth $(B)$ becoming sufficiently large to result in an additional strongly velocity matched interactions. In Fig. 1, the dashed vertical lines identify these points $\left(B / 2=\left|\Delta f_{a b c d}\right|\right)$. As the WDM bandwidth is increased, a higher number of strong intermodal nonlinear contributions are accumulated.

\section{LINEAR MODE COUPLING}

The analysis in section II was performed assuming the weak coupling regime. However, in the literature, the mode coupling values of fabricated FMFs range from $-50 \mathrm{~dB} / 100 \mathrm{~m}$ to $-40 \mathrm{~dB} / 100 \mathrm{~m}$ for fibres with step-index or graded-index profiles [14],[15], going up to $-28 \mathrm{~dB} / 100 \mathrm{~m}$ for coupled multicore fibres [16] and $-7 \mathrm{~dB} / 100 \mathrm{~m}$ for ring-index fibres [17].

To introduce linear mode coupling, we use a numerical mode coupling model which divides the fibre in multiple sections of length $L_{s}$, each with a constant random displacement of the core centre position [7],[8]. The model considers a random displacement of the radial and azimuthal coordinates. The mode coupling strength $(X T)$ is quantified as $X T_{m}=\sum_{n \neq m} P_{n} / P_{m}$ where $P_{n}$ is the power of mode $n$, after a given segment under test, when only mode $m$ was launched. If $X T_{m}$ is equal to $0 \mathrm{~dB}$, then half of the power launched in mode $m$ has been transferred to other modes.

Fig. 2 shows the mode coupling strength averaged over the azimuth displacement, as a function of the normalised radial displacement, for a 4 non-degenerated LP mode fibre. Note that, coupling strengths are calculated considering degenerate modes such as $\mathrm{LP}_{11 \mathrm{a}}$ and $\mathrm{LP}_{11 \mathrm{~b}}$ as one mode. In Fig. 2, it can be seen that the mode coupling strength only depends significantly on the mode being considered for displacements higher than $1 \%$. Such higher coupling for $\mathrm{LP}_{02}$ and $\mathrm{LP}_{21}$ can be explained noting they belong to the same mode group. Moreover, $X T_{L P_{21}}<$ $X T_{L P_{02}}$ for any displacement in Fig. 2 because any power launched in $\mathrm{LP}_{21 \mathrm{a}}$ couples preferentially with $\mathrm{LP}_{21 \mathrm{~b}}$ (and viceversa) and in the second place to $\mathrm{LP}_{02}$. Given the higher values of $X T_{L P_{02}}$, we define $L_{c}$ for this mode. Note that $X T_{m}$ values above $+10 \mathrm{~dB}$ mean that almost all power launched in mode $m$ has been transferred to other modes.

In the next sections, the impact of linear mode coupling on the statistics of the group delays and on the intermodal nonlinear interactions efficiency will be analysed. 


\section{GROUP Delays Statiscs WITH INTERMEDIATE COUPLING}

As already explained, for FMFs, the phase matching of intermodal FWM is achieved by balancing the two dispersive processes present, the mode group delay and the chromatic dispersion. Therefore, it can be anticipated that the impact of linear mode coupling on the group delays statistics will have consequently a significant impact on the efficiency of intermodal FWM.

In this section, we will evaluate the impact of linear mode coupling on the statistics of the GDs of the PMs considering the same fibre used in section II, characteristics in Table 1. The $X T_{L P_{02}}$ value was varied from -50 to $0 \mathrm{~dB} / 100 \mathrm{~m}$ by varying the amplitude of the variation in lateral section offset assuming a section length of $100 \mathrm{~m}$. This range fully covers the range of coupling values presented in the literature [14]-[17]. Finally, the GDs of the PMs were calculated through direct numerical solution of the coupled-mode equations describing the linear propagation [7],[8].

In the intermediate coupling regime, the statistics of the GDs, we shown in [10] that at least for fibres guiding up to 4 non-degenerate LP modes, the standard deviation of the GDs in FMFs can be approximated by:

$$
\sigma_{g d}=\sqrt{2} \sigma_{\tau} L_{c}\left(e^{-L / L_{c}}+\frac{L}{L_{c}}-1\right)^{1 / 2}
$$

where $\sigma_{\tau}$ is the standard deviation of the uncoupled GDs per unit length and $L_{c}$ is the correlation length defined as the length for which $\left[P_{m}\left(L_{c}\right)-\sum_{v \neq m} P_{v}\left(L_{c}\right)\right] / \sum_{n} P_{n}\left(L_{c}\right)=e^{-2}$, where $P_{v}$ is the power of mode $v$ and mode $m$ is the mode presenting higher coupling strength. Using equation (3), it can be shown that for negligible coupling (less than $-40 \mathrm{~dB} / 100 \mathrm{~m}$, for typical transmission distances), $\sigma_{g d}$ scales approximately linearly with distance. However, above this value FMFs are typically operating in intermediate coupling regime, and increasing $X T_{L P_{02}}, \sigma_{g d}$ gradually converges to the strong coupling regime ( $\sigma_{g d}$ becames directly proportional to $L^{1 / 2}$ ).

Using equation (3), it can be shown that by increasing the mode coupling the variance of the mode group delays is decreased, this is, the mode group delays became more similar and the maximum differential delay is reduced. However, at the same time, the range of values that a given coupled group delay can take increases.

Fig. 3 shows the PDF of the ordered GDs $\left(\tau_{m}, \tau_{1} \leq \tau_{2} \leq\right.$ $\cdots \leq \tau_{6}$ ), normalized by the $\sigma_{g d}$ of the PMs, after $1000 \mathrm{~km}$ for two different coupling values, overlapped with the analytical joint PDF derived for the strong coupling regime [2]. Note that the normalization factor $\left(\sigma_{g d}\right)$ depends on the $X T_{L P_{02}}\left(L_{c}\right)$ value, see Eq. (3). Fig. 3 (a) shows that for $-30 \mathrm{~dB} / 100 \mathrm{~m}$ the GDs of the PMs vaguely resemble the GDs of the LP modes given the impulse-like PDF of $\tau_{2}$ ("LP $\mathrm{P}_{11 \mathrm{a}}$ ") and $\tau_{3}$ ("LP $11 \mathrm{~b}$ "). Further results for lower coupling values shown that all GDs present impulse-like PDFs. In Fig. 3 (b), for $-20 \mathrm{~dB} / 100$ m, the match between the simulated PDFs and the analytical PDF for strong

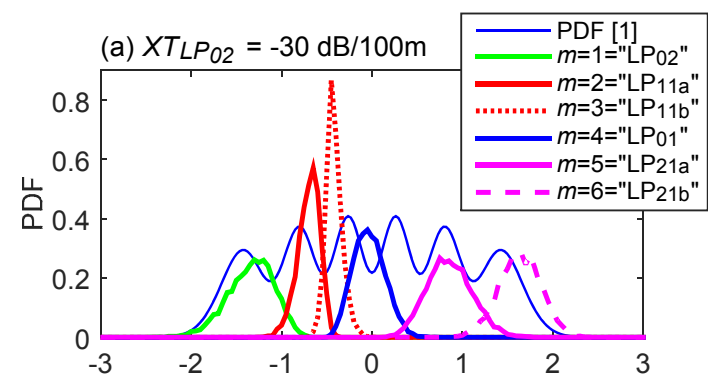

(b) $X T_{L P_{02}}=-20 \mathrm{~dB} / 100 \mathrm{~m}$

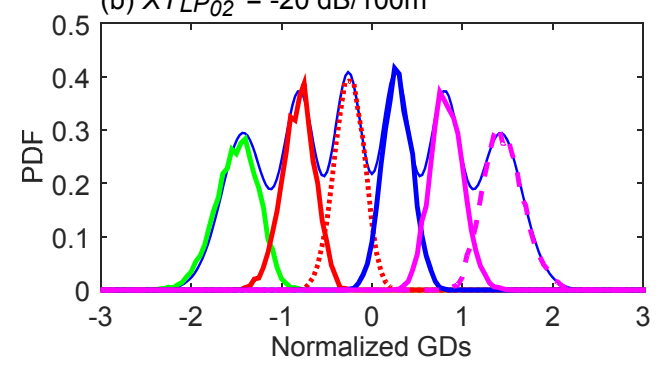

Fig. 3: Probability density function of the ordered normalized GDs $\left(\tau_{\mathrm{m}} / \sigma_{\mathrm{gd}}\right)$, obtained through simulation after $1000 \mathrm{~km}$, with different $\mathrm{XT}_{\mathrm{LP}_{02}}$ values.

coupling is good, even though the GDs have been normalized by different factors.

In this section, we shown that in the presence of linear mode coupling the average value of the GDs became similar as the variance of the GDs decreases, therefore phase matching (as in Section II) should occur for smaller frequency separations. However, given that the range of values that a given GD can take increases the efficiency of the nonlinear interactions may be reduced.

\section{INTERMODAL FWM WITH INTERMEDIATE COUPLING}

The analysis in section III shown that intermediate coupling strength can have a significant impact on the statistics of the group delays. Thus, the analysis of intermodal FWM developed in section II has to be performed again assuming the intermediate coupling regime, as these is the most common regime in practice. The majority of the FMFs presented up now [20] show higher crosstalk, ranging from $-50 \mathrm{~dB} / 100 \mathrm{~m}$ to $-40 \mathrm{~dB} / 100 \mathrm{~m}$, and new fibres presenting considerably stronger crosstalk have been reported [21] $(-7 \mathrm{~dB} / 100 \mathrm{~m})$.

In the strong coupling regime, the nonlinear interactions between the modes can be averaged with respect to the random linear evolution of the field within each mode, since the linear evolution is significantly faster than the nonlinear evolution, as shown in [5],[6]. In this case, for $M$ modes with 2 polarizations, the nonlinear terms average to $-\mathrm{j} \Gamma|\vec{E}|^{2} \vec{E}$, where $\Gamma=4 / 3$. $(2 M) /(2 M+1) \cdot \bar{\gamma}$ with $\bar{\gamma}$ equal to the average nonlinear coefficient [6]. Moreover, the $\Gamma$ coefficient can be introduced to (2) in place of $\gamma_{a b c d}$.

In this paper, the nonlinear noise power was calculated for a range of different linear coupling strengths ranging from the strong to the weak coupling regimes using a numerical model which divides the fibre in multiple sections of length, each with 
a constant random displacement of the core centre position [7],[8]. The coupling strength $(X T)$ was quantified as the ratio between the power lost to the other modes from the $\mathrm{LP}_{01}$ mode and the power that remains in the $\mathrm{LP}_{01}$ mode. The nonlinear signal field $E_{d s}$ generated after a length $L$ is found by adding (1) over all fiber segments, while the interfering waves $a p, b q, c r$ are linearly propagated (attenuation, dispersion and linear mode coupling). As for dispersion managed single mode fibre systems, power depletion due to the FWM and higher-order mixing terms are neglected [22]. Finally, the total nonlinear noise power generated at the centre of the WDM versus the WDM bandwidth is obtained by summing over the frequency offsets that satisfy $\omega_{p}+\omega_{q}=\omega_{r}+\omega_{s}$.

Fig. 4 shows the nonlinear noise power generated at the centre of the WDM band versus the WDM bandwidth for the fibre presented in Table I and for different $X T$ values ranging from $-70 \mathrm{~dB} / 100 \mathrm{~m}$ to $-20 \mathrm{~dB} / 100 \mathrm{~m}$. The $X T$ value was set by varying the amplitude of the random variation in lateral section offset assuming a section length of $10 \mathrm{~m}$ (smaller section lengths generated similar results). The simulation results in Fig. 4 lay between two analytical lines obtained with (2) using: the ordinary fibre nonlinear coefficients (for zero, or weak mode coupling) [5], dotted line, and using the average nonlinear coefficients derived in [6] for strong mode coupling, dashed line.

It can be noted in Fig. 4 that the rate of decrease of the nonlinear noise as the coupling strength is increased is higher for higher bandwidths than for smaller bandwidths. This observation is in line with the conclusions of Section IV, with increasing coupling strength, the maximum difference between the coupled GDs is decreased, and thereby the phase matching is achieved for lower frequency separations. For small values of $X T,-70$ and $-60 \mathrm{~dB} / 100 \mathrm{~m}$ the steps associated with the intermodal interactions of $\mathrm{LP}_{01}$ with $\mathrm{LP}_{02}$ and $\mathrm{LP}_{21 \mathrm{a}} / \mathrm{LP}_{21 \mathrm{~b}}$, become smooth, but the step associated with $\mathrm{LP}_{11 \mathrm{a}} / \mathrm{LP}_{11 \mathrm{~b}}$ remains unchanged. Again, as explained in Section IV, the range of values that the coupled of GDs can take increase, thereby smoothing these step-like transitions. Moreover, the different impact of coupling on different modes is in line with the asymmetries on the coupling strength between pairs of modes from the same mode groups (stronger) and from different mode groups (weaker) (modes $\mathrm{LP}_{02}$ and $\mathrm{LP}_{21 \mathrm{a}} / \mathrm{LP}_{21 \mathrm{~b}}$ belong to the same mode group), as discussed in Section III. Increasing XT up to $-40 \mathrm{~dB} / 100 \mathrm{~m}$, smooths the step associated with intermodal interactions of $\mathrm{LP}_{01}$ with $\mathrm{LP}_{02}$. Furthermore, increasing $X T$ above $-20 \mathrm{~dB} / 100 \mathrm{~m}$ reduces nonlinear noise power below the $\mathrm{LP}_{01}$ intra-mode nonlinear noise power in the absence of linear coupling which was used to normalize the results. In the limit, strongly coupling all modes, using unitary matrices every $10 \mathrm{~m}$ (and shorter steps, as verified), the nonlinear noise power matches the analytical results (dashed line) obtained using (2) and the average nonlinear coefficients in [6].

Finally, we further analyse the rate of decrease of the nonlinear noise as a function of bandwidth for increasing coupling strength. Specifically, we focus on the multi-channel interference (MCI), as defined in [23], considering a channel bandwidth of $1 \mathrm{GHz}$. Fig. 5 shows the MCI normalized by the MCI obtained in the absence of linear mode coupling as a function of WDM bandwidth for coupling strengths ranging between -70 and $-40 \mathrm{~dB} / 100 \mathrm{~m}$. From Fig. 5 it can be seen that

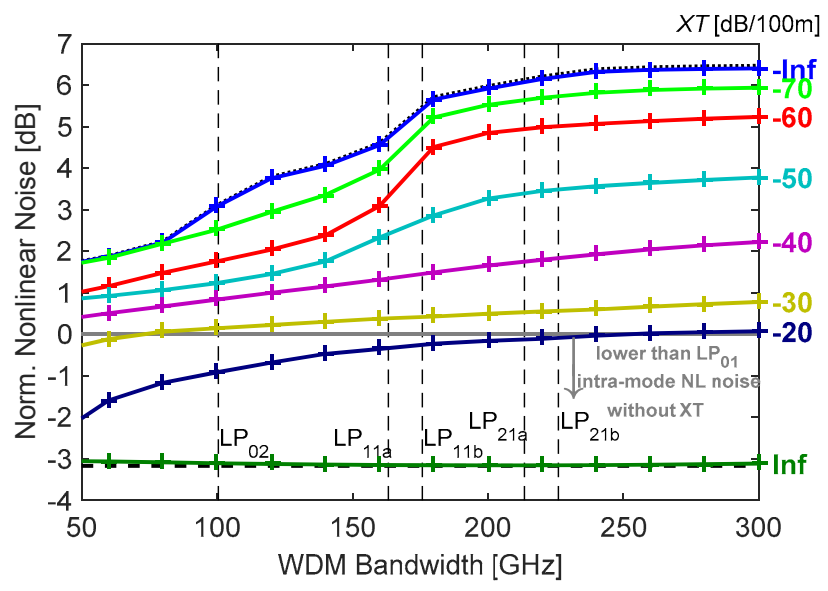

Fig. 4. Total nonlinear noise power in the $\mathrm{LP}_{01}$ mode as a function of WDM bandwidth showing analytical predictions from strong (dashed) and weak (dotted) regimes along with numerical simulations (solid) for different mode coupling strengths (colors).

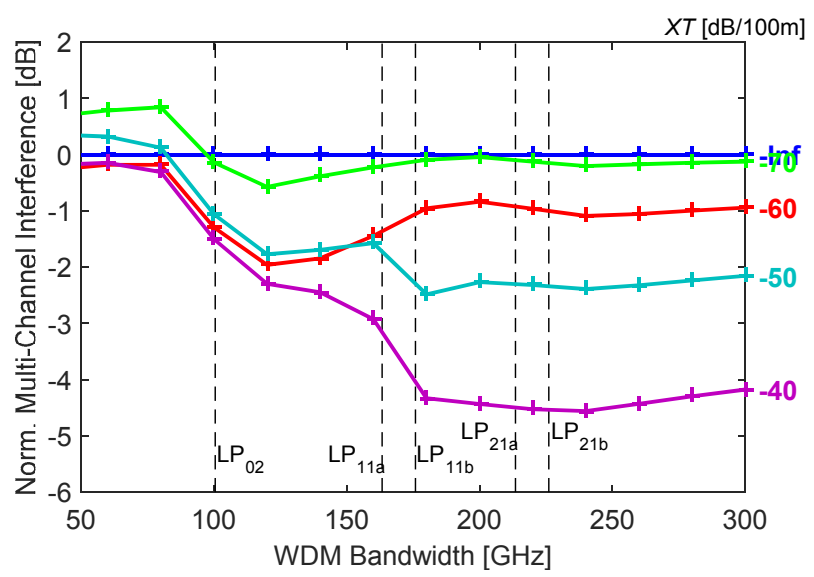

Fig. 5. Multi-channel interference normalized by the multi-channel interference obtained in the absence of linear mode coupling as a function of WDM bandwidth for different mode coupling strengths (colors).

for bandwidths narrow than $100 \mathrm{GHz}$, MCI either increases or slightly decreases with increasing coupling strength. On the other side, for bandwidths wider than $100 \mathrm{GHz}$, MCI decreases significantly with increasing coupling strengths. Therefore, Fig. 5 allows to verify that the frequency separation required for phase matching for a given set of modes is reduced with increasing coupling strength.

\section{CONCLUSIONS}

In this paper, we have investigated the impact of linear mode coupling on the efficiency of intermodal FWM in FMFs stressing its intrinsic connection to the mode group delay statistics. The results presented allowed to conclude that for the crosstalk values shown by the majority of FMFs (from -50 to $-20 \mathrm{~dB} / 100 \mathrm{~m}$ ), the nonlinear noise is not accurately estimated by either the weak linear coupling regime or the strong coupling regime. However, the overall conclusion that the stronger coupling reduces nonlinear noise power remains valid. More 
importantly, we have assessed the level of intermediate coupling required to minimize nonlinear noise in coupled fewmode propagation below that of uncoupled single-mode propagation. We observed a reduction of nonlinear noise below that of uncoupled single-mode propagation for linear coupling values above $-20 \mathrm{~dB} / 100 \mathrm{~m}$.

\section{ACKNOWLEDGMENT}

This work has been partially supported by the European Union (Grants 619732-INSPACE, 654809-HSPACE, 659950INVENTION, and 627545-SOLAS), and by the EPSRC (Grant EP/L000091/1-PEACE). The research data supporting this publication is available at http://dx.doi.org/10.17036/e2d3ab9b4ed0-4fc2-88e0-c9c5b029c4d5.

\section{REFERENCES}

[1] D. Marcuse, Theory of Dielectric Optical Waveguides, 2nd ed. New York: Academic, 1991.

[2] K. Ho, et al., "Statistics of group delays in multimode fiber with strong mode coupling," J. Lightw. Technol., 29(21), p 3119, 2011.

[3] R.-J. Essiambre, et al., "Inter-modal nonlinear interactions between well separated channels in spatially-multiplexed fiber transmission," in Proc. ECOC, p. Tu.1.C.4, 2012.

[4] F. Poletti, et al., "Description of ultrashort pulse propagation in multimode optical fibers," J. Opt. Soc. Am. B 25, p.1645, 2008.

[5] S. Mumtaz, et al., "Nonlinear Propagation in Multimode and Multicore Fibers: Generalization of the Manakov Equations," J. Lightw. Technol., 31(3), p.398, 2013.

[6] A. Mecozzi, et al., "Nonlinear propagation in multi-mode fibers in the strong coupling regime," Opt. Express 20, p.11673, 2012.

[7] F. Ferreira, et al., "Nonlinear Semi-Analytical Model for Simulation of Few-Mode Fiber Transmission," Photon. Technol. Lett., 24(4), p.240, 2012.

[8] F. Ferreira, et al., "Semi-Analytical Model for Linear Modal Coupling in Few-Mode Fiber Transmission," in Proc. ICTON, p. Th.A1.5, 2012.
[9] F. Ferreira, et al., "Advantages of Strong Mode Coupling for Suppression of Nonlinear Distortion in Few-Mode Fibers," in Proc. OFC, p. Tu2E.3, 2016.

[10] F. Ferreira, et al. "Few-Mode Fibre Group-Delays with Intermediate Coupling," in Proc. ECOC, 2015.

[11] Y. Xiao, et al., "Theory of intermodal four-wave mixing with random linear mode coupling in few-mode fibers," Opt. Express 22, p. 32039 2014.

[12] A. Ellis, et al., "Expressions for the nonlinear transmission performance of multi-mode optical fiber," Opt. Express 21, p. 22834, 2013.

[13] G. Rademacher, et al., "Analytical description of cross modal nonlinear interaction in mode multiplexed multi-mode fibers," Photon. Technol. Lett., 24(21), p. 1929, 2012.

[14] L. Grüner-Nielsen, et al., "Few Mode Transmission Fiber With Low DGD, Low Mode Coupling, and Low Loss," J. Lightw. Technol., Vol. 30, no. 23, p. 3693, 2012.

[15] T. Mori, et al., "Low DMD Four LP Mode Transmission Fiber for Wideband WDM-MIMO System," in Proc. OFC, p. OTh3K.1, 2013.

[16] R. Ryf, et al., "Space-division multiplexed transmission over 4200-km 3core microstructured fiber," in Proc. OFC, p. PDP5C.2, 2012.

[17] N. Fontaine, et al., "Experimental investigation of crosstalk accumulation in a ring-core fiber," in Proc. PSSTMS, p. TuC4.2, 2013.

[18] F. Ferreira, et al., "Design of few-mode fibers with M-modes and low differential mode delay," J. Lightw. Technol., 32(3), p.353, 2014.

[19] O. Sinkin, et al., "Impact of broadband four-wave mixing on system characterisation," in Proc. OFC, p. OTh3G, 2013.

[20] L. Grüner-Nielsen, et al., "Few mode transmission fiber with low DGD, low mode coupling, and low loss," J. Lightw. Technol., 30(23), p. 3693, 2012.

[21] N. Fontaine, et al., "Experimental investigation of crosstalk accumulation in a ring-core fiber," in Proc. PSSTMS, p. TuC4.2, 2013.

[22] K. Inoue, "Phase-mismatching characteristic of four-wave mixing in fiber lines with multistage optical amplifiers," Opt. Lett. 17, p.801, 1992.

[23] P. Poggiolini, "The GN Model of Non-Linear Propagation in Uncompensated Coherent Optical Systems," J. Lightw. Technol., 30(24), p. $3857,2012$. 